\title{
MULTIALTERNATING GRADED POLYNOMIALS AND GROWTH OF POLYNOMIAL IDENTITIES
}

\author{
ELI ALJADEFF AND ANTONIO GIAMBRUNO
}

(Communicated by Harm Derksen)

\begin{abstract}
Let $G$ be a finite group and $A$ a finite dimensional $G$-graded algebra over a field of characteristic zero. When $A$ is simple as a $G$-graded algebra, by means of Regev central polynomials we construct multialternating graded polynomials of arbitrarily large degree non-vanishing on $A$. As a consequence we compute the exponential rate of growth of the sequence of graded codimensions of an arbitrary $G$-graded algebra satisfying an ordinary polynomial identity. If $c_{n}^{G}(A), n=1,2, \ldots$, is the sequence of graded codimensions of $A$, we prove that $\exp ^{G}(A)=\lim _{n \rightarrow \infty} \sqrt[n]{c_{n}^{G}(A)}$, the $G$-exponent of $A$, exists and is an integer. This result was proved by the authors and D. La Mattina in 2011 and by the second author and D. La Mattina in 2010 in the case $G$ is abelian.
\end{abstract}

\section{INTRODUCTION}

Let $F$ be a field of characteristic zero and $A$ an $F$-algebra graded by a finite group $G$. We shall assume throughout that $A$ is a PI-algebra; i.e., it satisfies an ordinary polynomial identity. The graded polynomial identities satisfied by $A$ and their growth have been extensively studied in the last years in an effort to develop a general theory that generalizes the theory of ordinary polynomial identities.

For instance, in analogy with a basic result in Kemer's theory (see [15]), it was recently proved in 2 (and independently in [20, for $G$ abelian) that if $A$ is a finitely generated algebra, then $A$ satisfies the same graded identities as a finite dimensional graded algebra. As a consequence one can reduce the study of an arbitrary $G$-graded PI-algebra to that of the Grassmann envelope of a finite dimensional $\mathbb{Z}_{2} \times G$-graded algebra.

Also, in analogy with the results in [11] and [12] concerning the existence of the exponent of a PI-algebra, in [1] and [9] it was shown that when $G$ is an abelian group $\exp ^{G}(A)$, the graded exponent of $A$ exists and is an integer.

Here we focus on the growth of the graded codimensions of $A$. Recall that if $P_{n}^{G}$ is the space of multilinear graded polynomials of degree $n$ and $\operatorname{Id}^{G}(A)$ is the ideal of graded identities of $A$, then $c_{n}^{G}(A)$, the $n$th $G$-codimension of $A$, measures the dimension of $P_{n}^{G} \bmod \operatorname{Id}^{G}(A)$.

Received by the editors July 22, 2011 and, in revised form, December 5, 2011.

2010 Mathematics Subject Classification. Primary 16R50, 16P90, 16R10, 16W50.

Key words and phrases. Graded algebra, polynomial identity, growth, codimensions.

The first author was supported by the Israel Science Foundation (grant No. 1283/08) and by the E. Schaver Research Fund.

The second author was partially supported by MIUR of Italy. 
It was known that the sequence $c_{n}^{G}(A), n=1,2, \ldots$, is exponentially bounded ([10]), but only recently its exponential rate of growth was captured in the case $G$ is an abelian group. In fact in [1] for finitely generated algebras and in 9] in general, it was shown that if $A$ is any $G$-graded PI-algebra and $G$ is a finite abelian group, then $\exp ^{G}(A)=\lim _{n \rightarrow \infty} \sqrt[n]{c_{n}^{G}(A)}$ exists and is an integer called the $G$-exponent of $A$. Moreover, the $G$-exponent can be explicitly computed and equals the dimension of a suitable finite dimensional semisimple graded algebra related to $A$.

In this note we shall extend the above result by proving that the $G$-exponent exists and is an integer in the case of arbitrary (non-necessarily abelian) groups $G$. We notice that the proof given in [9] also works when $G$ is a non-abelian group modulo two basic ingredients that we manage to prove here. The first result says that the multiplicities in the $n$th graded cocharacter of $A$ are polynomially bounded, and the second is of independent interest and consists of the construction of suitable multialternating graded polynomials non-vanishing in a finite dimensional $G$-graded algebra which is simple as a graded algebra.

Throughout the paper $F$ will be a field of characteristic zero and $A$ an associative $F$-algebra satisfying a non-trivial polynomial identity. We assume that $A$ is graded by a finite group $G=\left\{g_{1}=1, g_{2}, \ldots, g_{s}\right\}$, and we let $A=\bigoplus_{g \in G} A_{g}$ be its decomposition into a sum of homogeneous components.

\section{Multialternating polynomials on $G$-Simple Algebras}

We start by introducing some standard notation.

We let $F\langle X, G\rangle$ be the free associative $G$-graded algebra of countable rank over $F$. The set $X$ decomposes as $X=\bigcup_{i=1}^{s} X_{g_{i}}$, where the sets $X_{g_{i}}=\left\{x_{1, g_{i}}, x_{2, g_{i}}, \ldots\right\}$ are disjoint and the elements of $X_{g_{i}}$ have homogeneous degree $g_{i}$. The algebra $F\langle X, G\rangle$ is endowed with a natural $G$-grading $F\langle X, G\rangle=\bigoplus_{g \in G} \mathcal{F}_{g}$, where $\mathcal{F}_{g}$ is the subspace spanned by the monomials $x_{i_{1}, g_{j_{1}}} \cdots x_{i_{t}, g_{j_{t}}}$ of homogeneous degree $g=g_{j_{1}} \cdots g_{j_{t}}$.

Recall that a graded polynomial $f \in F\langle X, G\rangle$ is a graded (polynomial) identity of $A$ if $f$ vanishes under all graded substitutions $x_{i, g}=a_{g} \in A_{g}$. Also let $\operatorname{Id}^{G}(A)=$ $\{f \in F\langle X, G\rangle \mid f \equiv 0$ on $A\}$ be the ideal of graded identities of $A$.

We say that an algebra $A$ is $G$-graded simple if $A$ is a $G$-graded algebra which is simple as a graded algebra.

Let $A$ be a finite dimensional $G$-graded simple algebra over an algebraically closed field of characteristic zero. The purpose of this section it to produce nonidentity $G$-graded polynomials with arbitrary many alternating sets of variables which correspond to the homogeneous components of $A$ and with a bounded number of extra variables.

A key ingredient in the construction of these polynomials is a presentation of any $G$-graded simple algebra as a tensor product of two types of $G$-graded simple algebras, namely a twisted group algebra (with fine grading) and a matrix algebra with an elementary grading. Here is the precise statement. It is due to Bahturin, Sehgal and Zaicev.

Theorem 1.1 ([4]). Let $A$ be a finite dimensional G-graded simple algebra. Then there exists a subgroup $H$ of $G$, a 2-cocycle $\alpha: H \times H \rightarrow F^{*}$ where the action of $H$ on $F$ is trivial, an integer $r$ and an $r$-tuple $\left(g_{1}, g_{2}, \ldots, g_{r}\right) \in G^{r}$ such that $A$ is $G$-graded isomorphic to $\Lambda=F^{\alpha} H \otimes M_{r}(F)$ where $\Lambda_{g}=\operatorname{span}_{F}\left\{\pi_{h} \otimes e_{i, j} \mid g=g_{i}^{-1} h g_{j}\right\}$. Here 
$\pi_{h} \in F^{\alpha} H$ is a representative of $h \in H$ and $e_{i, j} \in M_{r}(F)$ is the $(i, j)$ elementary matrix.

In particular, the idempotents $1 \otimes e_{i, i}$ as well as the identity element of $A$ are homogeneous of degree $e \in G$.

Let $t_{1}, \ldots, t_{s}>0$ be integers and, for $i=1, \ldots s$, define

$$
X_{g_{i}}^{j}=\left\{x_{1, g_{i}}^{j}, \ldots, x_{m_{i}, g_{i}}^{j}\right\} \subseteq X_{g_{i}}, 1 \leq j \leq t_{i},
$$

as $t_{i}$ distinct sets consisting of $m_{i} \geq 0$ variables, each of homogeneous degree $g_{i}$. Also let $Y \subseteq \bigcup_{i=1}^{s} X_{g_{i}}$ be another set of homogeneous variables disjoint from the previous sets.

In addition, let $f=f\left(X_{g_{1}}^{1}, \ldots, X_{g_{1}}^{t_{1}}, \ldots, X_{g_{s}}^{1}, \ldots, X_{g_{s}}^{t_{s}}, Y\right) \in F\langle X, G\rangle$ be a multilinear graded polynomial in the variables from the sets $X_{g_{i}}^{j}$ and $Y, 1 \leq i \leq s$ and $1 \leq j \leq t_{i}$.

This section is devoted to the proof of the following.

Theorem 1.2. Let $F$ be an algebraically closed field of characteristic zero and $A$ be a finite dimensional $G$-graded simple algebra over $F$. For any $t \geq 1$, there exist integers

and a G-graded polynomial

$$
2 t \leq t_{1}, \ldots, t_{s} \leq 2 t|G|
$$

$$
f_{t}\left(X_{G}^{\left(t_{1}, \ldots, t_{s}\right)} ; Y\right)=f_{t}\left(X_{g_{1}}^{1}, \ldots, X_{g_{1}}^{t_{1}}, X_{g_{2}}^{1}, \ldots, X_{g_{2}}^{t_{2}}, \ldots, X_{g_{s}}^{1}, \ldots, X_{g_{s}}^{t_{s}} ; Y\right)
$$

such that:

(1) $f_{t}\left(X_{G}^{\left(t_{1}, \ldots, t_{s}\right)} ; Y\right)$ is not an identity of $A$; in particular, it has an evaluation in $A$ of the form $1 \otimes e_{i, j}$.

(2) The cardinality of $Y$ depends on the order of $G$ and the dimension of $A$ and not on the parameter $t$. In particular, the cardinality of $Y$ is bounded.

(3) For every $j$ and $g \in G,\left|X_{g}^{j}\right|=\operatorname{dim} A_{g}$.

(4) $f_{t}\left(X_{G}^{\left(t_{1}, \ldots, t_{s}\right)} ; Y\right)$ is alternating on each of the sets $X_{g}^{j}$.

In view of the theorem above we claim that it is sufficient to construct $G$-graded polynomials, which are non-identities of $A$ and correspond to the cyclic subgroups of $G$.

In order to make the statement precise, let $g$ be any element of $G$ and let $S=\langle g\rangle$ be the subgroup it generates. We denote by $d$ the order of $S$.

Proposition 1.3. It is sufficient to construct, for any integer $t \geq 1$, a G-graded polynomial (non-identity of $A$ )

$$
f_{t, g}\left(X_{S} ; Y_{S}\right)=f_{t, g}\left(X_{e}^{1}, \ldots, X_{e}^{2 t}, X_{g}^{1}, \ldots, X_{g}^{2 t}, \ldots, X_{g^{d-1}}^{1}, \ldots, X_{g^{d-1}}^{2 t} ; Y_{S}\right)
$$

where

(1) $\left|Y_{S}\right| \leq r-1$ ( $r$ is the cardinality of the tuple which provides the elementary grading on $A$ ).

(2) For every $i=1, \ldots, 2 t$ and every $0 \leq j \leq d-1$, we have that $\left|X_{g^{j}}^{i}\right|=$ $\operatorname{dim} A_{g^{j}}$.

(3) $f_{t, g}\left(X_{S} ; Y_{S}\right)$ is alternating on the set $X_{g^{j}}^{i}$ for every $i=1, \ldots, 2 t$ and every $0 \leq j \leq d-1$.

(4) $f_{t, g}\left(X_{S} ; Y_{S}\right)$ admits a non-zero G-graded evaluation on $A$ of the form $\pi_{g^{l}} \otimes$ $e_{1, r}$. 
Remark 1.4. Clearly, by adding an extra variable if necessary, we may assume that the value of the polynomial above is $1 \otimes e_{1,1}$.

Proof. Indeed, having constructed the polynomials $f_{t, g}=f_{t, g}\left(X_{S} ; Y_{S}\right)$ above, the required polynomials are given by

$$
f_{t}\left(X_{G}^{\left(t_{1}, \ldots, t_{s}\right)} ; Y\right)=\prod_{g \in G} f_{t, g}\left(X_{S} ; Y_{S}\right),
$$

where $|Y|=\sum_{g \in G} Y_{S}$.

Consider the subalgebra $A_{S}=\bigoplus_{i=0}^{d-1} A_{g^{i}} \subseteq A$. By [17, Theorem 1.6] (see also [16. Theorem 18.13]), it is semisimple, and so it decomposes into the direct sum of $S$-graded simple algebras

$$
A_{S} \cong B_{1} \oplus B_{2} \oplus \cdots \oplus B_{l} .
$$

It follows from Bahturin, Sehgal and Zaicev's result that for every $i=1, \ldots, l$, there exist a subgroup $C_{i} \leq S$ and a $p_{i}$-tuple $\left(w_{i, 1}, \ldots, w_{i, p_{i}}\right)$ of elements in $S$ (which determines the elementary grading on $\left.M_{p_{i}}(F)\right)$ such that

$$
B_{i} \cong F C_{i} \otimes M_{p_{i}}(F)
$$

as $S$-graded algebras.

Notice that since $C_{i}$ is cyclic, $H^{2}\left(C_{i}, F^{*}\right)=0,1 \leq i \leq l$.

The structure of $A_{S}$ is given here up to an $S$-graded isomorphism. But we need more. In fact, in order to "bridge" the $S$-simple components $B_{i}$ by elements of $A$, we need to realize the algebra $A_{S}$ as a subalgebra of $A$ in terms of its presentation (i.e. with the terminology of Theorem 1.1). Here is the precise statement.

First we make a definition: for a homogeneous subspace $D$ of $A$ we define weight $(D)=\left\{g_{i} \in G \mid D \cap A_{g_{i}} \neq 0\right\}$.

Proposition 1.5. With the above notation:

(1) For every $i=1, \ldots, l, B_{i} \cong F C_{i} \otimes M_{p_{i}}(F)$, where $C_{i}=H^{g_{j_{i}}} \cap S$.

(2) In terms of the presentation of $A$, after a possible permutation of the $r$ tuple $\left(g_{1}, \ldots, g_{r}\right)$ (the tuple which provides the elementary grading in the presentation of $A)$, we have

$$
B_{i}=\operatorname{span}\left\{\pi_{h} \otimes e_{u, v} \in A_{S} \mid p_{1}+p_{2}+\cdots+p_{i-1}+1 \leq u, v \leq p_{1}+p_{2}+\cdots+p_{i}\right\} .
$$

In particular, $p_{1}+p_{2}+\cdots+p_{l}=r$.

(3) For every $i=1, \ldots, l$,

$$
B_{i}=B_{i, 1} \oplus \cdots \oplus B_{i, c_{i}}
$$

(a direct sum of vector spaces that we shall call "pages"), where

(a) $B_{i, k}=\operatorname{span}\left\{\pi_{h_{u, v}} \otimes e_{u, v} \in A_{S} \mid p_{1}+p_{2}+\cdots+p_{i-1}+1 \leq u, v \leq\right.$ $\left.p_{1}+p_{2}+\cdots+p_{i}\right\}$ (for any pair $(u, v)$ as above we choose a suitable $\left.h=h_{u, v} \in H\right)$.

(b) Any homogeneous component of $B_{i}$ is "concentrated" in a unique page. More precisely, weight $\left(B_{i, k}\right) \cap$ weight $\left(B_{i, l}\right)=\emptyset$ if $k \neq l$.

Before proving the proposition, let us show how to construct the polynomial $f_{t, g}\left(X_{S} ; Y_{S}\right)$. 
For each "page" $B_{i, k}$ we construct a Regev polynomial $f_{2 p_{i}^{2}}^{k}$ (see [8]) with $2 p_{i}^{2}$ variables whose homogeneous degrees are as the homogeneous degrees of $B_{i, k}$, and we let

$$
f_{i}=f_{2 p_{i}^{2}}^{1} f_{2 p_{i}^{2}}^{2} \cdots f_{2 p_{i}^{2}}^{c_{i}}
$$

Now, the evaluation of $f_{2 p_{i}^{2}}^{k}$ on a basis of $B_{i, k}$ gives an element of the form $\pi_{b_{k}} \otimes 1_{p_{i} \times p_{i}}$, where $\pi_{b_{k}}$ is a trivial unit of $F C_{i}$. This is a slight abuse of notation since in fact the identity matrix $1_{p_{i} \times p_{i}}$ is located in the block diagonal between rows $p_{1}+p_{2}+\cdots+p_{i-1}+1$ and $p_{1}+p_{2}+\cdots+p_{i}$.

Remark 1.6. This is the place where we use the fact that $C_{i}$ is a cyclic group. Indeed, the evaluation of a Regev polynomial on the space $B_{i, k}$ has the same effect as the evaluation on $p_{i} \times p_{i}$ matrix algebra where all monomials are multiplied by the same trivial unit which is obtained as the product of commuting trivial units of $F C_{i}$.

From the construction of $f_{i}$ we see that it has an evaluation of the form $\pi_{b_{i}^{\prime}} \otimes$ $1_{p_{i} \times p_{i}}$, where $\pi_{b_{i}^{\prime}}$ is a trivial unit of $F C_{i}$. Note that since the homogeneous degrees of the spaces $B_{i, k}$ are disjoint for different $k$, the polynomial $f_{i}$ is alternating (in particular) on sets of $g^{\nu}$-variables, where $g^{\nu} \in S$, of cardinality which is equal to the dimension of the $g^{\nu}$-homogeneous subspace of $B_{i}$.

In order to get arbitrarily many alternating sets we let $f_{i}^{t}$ be the product of $t$-copies (with disjoint sets of variables) of $f_{i}$. Clearly, the evaluation of $f_{i}^{t}$ on a basis of $B_{i}$ gives an element of the form $\pi_{a_{i}} \otimes 1_{p_{i} \times p_{i}}$, where $\pi_{a_{i}}$ is a trivial unit in $F C_{i}$.

Thus we have constructed a polynomial $f_{i}^{t}$ for any $B_{i}$. We can now "bridge" these polynomials and get a polynomial

$$
\phi_{S}=x_{0} f_{1}^{t} x_{1} f_{2}^{t} \cdots f_{l}^{t} x_{l+1} .
$$

We observe that with suitable evaluations on the $x$ 's the polynomial $\phi_{S}$ has an evaluation which is equal to $1 \otimes e_{1,1}$. But we are not done yet. We still need to alternate variables with the same homogeneous degrees in the different polynomials $f_{i}^{t}$ in a suitable way. More precisely, for every $s=1, \ldots, t$ we alternate all variables with the same homogeneous degrees which appear in the polynomials $f_{1}^{s}, f_{2}^{s}, \ldots, f_{l}^{s}$. Clearly, because of the bridging variables $x_{j}$, the resulting polynomial $f_{t, g}\left(X_{S} ; Y_{S}\right)$ admits the value $1 \otimes e_{1,1}$ and has the required form.

Let us now prove the proposition above. To set up the notation again we recall that $A$ has a presentation given by $F^{\alpha} H \otimes M_{r}(F)$ and the elementary grading is given by the $r$-th tuple $\left(g_{1}, g_{2}, \ldots, g_{r}\right)$. We let $S$ be the cyclic group generated by $g \in G$ and denote by $d$ its order. Let us introduce an equivalence relation on the index set $\{1, \ldots, r\}$ by setting $i \sim j$ if and only if $g_{i}^{-1} H g_{j} \cap S \neq \emptyset$. It is easy to see that this is indeed an equivalence relation, and we let

$$
\Omega_{1}, \ldots, \Omega_{l}
$$

be the equivalence classes. We may clearly assume (after reordering the tuple $\left.\left(g_{1}, g_{2}, \ldots, g_{r}\right)\right)$ that equivalent indices are adjacent to each other. In other words, we have integers $p_{1}, \ldots, p_{l}$ such that

$$
\begin{aligned}
& \Omega_{1}=\left\{1, \ldots, p_{1}\right\}, \Omega_{2}=\left\{p_{1}+1, \ldots, p_{1}+p_{2}\right\}, \ldots, \\
& \Omega_{l}=\left\{p_{1}+\cdots+p_{l-1}+1, \ldots, p_{1}+\cdots+p_{l}=r\right\} .
\end{aligned}
$$


We shall replace (as we may) elements from the $r$-tuple which represent the same right $H$-coset by the same representative.

Consider indices $i, j \in \Omega_{k}$. We know (by the definition of the equivalence relation) that there exists an $h \in H$ such that $g_{i}^{-1} h g_{j} \in S$. We claim that the number of elements $h \in H$ with that property depends on the index $k$ but not on $i$ and $j$. In other words, we claim the following.

Lemma 1.7. For every $i, j, k_{0} \in \Omega_{k},\left|g_{i}^{-1} H g_{j} \cap S\right|=\left|g_{k_{0}}^{-1} H g_{k_{0}} \cap S\right|$. Furthermore, the sets $g_{i}^{-1} H g_{j} \cap S$ are $g_{k_{0}}^{-1} H g_{k_{0}} \cap S$-cosets in $S$.

Proof. Take $z \in g_{i}^{-1} H g_{j} \cap S$. For different elements $q \in g_{j}^{-1} H g_{k_{0}} \cap S$, we obtain different elements $z q \in g_{i}^{-1} H g_{k_{0}} \cap S$ and hence $\left|g_{i}^{-1} H g_{k_{0}} \cap S\right| \geq\left|g_{j}^{-1} H g_{k_{0}} \cap S\right|$. On the other hand, taking inverses we see that $\left|g_{j}^{-1} H g_{k_{0}} \cap S\right|=\left|g_{k_{0}}^{-1} H g_{j} \cap S\right|$. With $i$, $j$ and $k_{0}$ being arbitrary, the first part of the lemma follows. For the second part, note that $g_{i}^{-1} H g_{i} \cap S$ and $g_{k_{0}}^{-1} H g_{k_{0}} \cap S$ are subgroups of the cyclic group $S$. By the first part of the proof, they have the same order and hence they coincide. Following the first part of the proof we see that $g_{i}^{-1} H g_{j} \cap S$ is a (right, and hence 2-sided (by commutativity)) $g_{i}^{-1} H g_{i} \cap S$-coset and the lemma is proved.

Now, observe that for $i=1, \ldots, l$, the algebra

$$
U_{i}=\operatorname{span}\left\{\pi_{h} \otimes e_{u, v} \in A_{S} \mid u, v \in \Omega_{i}\right\}
$$

is a direct summand of $A_{S}$, and so the proof of the proposition will be completed if we show that $U_{i}, i=1, \ldots, l$, is $S$-simple. To see this we exhibit an explicit presentation of $U_{i}$ as a $S$-simple algebra.

Fix $1 \leq k \leq l$ and $k_{0} \in \Omega_{k}$. Let $w_{i-n_{(k-1)}} \in S$ be a $g_{k_{0}}^{-1} H g_{k_{0}} \cap S$-coset representative of $g_{k_{0}}^{-1} H g_{i} \cap S$, where $p_{1}+\cdots+p_{k-1}+1 \leq i \leq p_{1}+\cdots+p_{k}$, and $n_{(k-1)}=p_{1}+\cdots+p_{k-1}$. Then the map

$$
\phi_{k}: \pi_{h} \otimes e_{i, j} \longmapsto w_{i-n_{(k-1)}} g_{i}^{-1} h g_{j} w_{j-n_{(k-1)}}^{-1} \otimes e_{i-n_{(k-1)}, j-n_{(k-1)}}
$$

determines an isomorphism of the $S$-graded algebra $U_{k}$ with $F\left(g_{k_{0}}^{-1} H g_{k_{0}} \cap S\right) \otimes$ $M_{p_{k}}(F)$. In the latter, the elementary grading is given by the $p_{k}$-tuple $\left(w_{1}, \ldots, w_{p_{k}}\right)$. Details are omitted. Finally, we note that Proposition 1.5 (b) follows from Lemma 1.7 (by ordering the coset's elements), and the proof of Proposition 1.5 is completed.

Remark 1.8. Note that since the group $g_{k_{0}}^{-1} H g_{k_{0}} \cap S$ is cyclic, its cohomology vanishes, and hence we may use group elements rather than representatives.

\section{Graded exponent}

Throughout, $F$ will be an algebraically closed field of characteristic zero and $A$ a $G$-graded PI-algebra over $F$ with $G=\left\{g_{1}=1, g_{2}, \ldots, g_{s}\right\}$ a finite group.

In this section we shall prove that the $G$-exponent of $A$ exists and is an integer. This result was proved in the case where $G$ is an abelian group in [1 for finitely generated algebras and in 9 in general. Here we shall closely follow that approach.

We start by recalling the general setting. The ideal of $G$-graded polynomial identities of $A$ is denoted $\operatorname{Id}^{G}(A)$. For every $n \geq 1$,

$$
P_{n}^{G}=\operatorname{span}_{F}\left\{x_{\sigma(1), g_{i_{\sigma(1)}}} \cdots x_{\sigma(n), g_{i_{\sigma(n)}}} \mid \sigma \in S_{n}, g_{i_{1}}, \ldots, g_{i_{n}} \in G\right\}
$$


is the space of multilinear $G$-graded polynomials in the homogeneous variables $x_{1, g_{i_{1}}}, \ldots, x_{n, g_{i_{n}}}, g_{i_{j}} \in G$. We construct the quotient space

$$
P_{n}^{G}(A)=\frac{P_{n}^{G}}{P_{n}^{G} \cap \operatorname{Id}^{G}(A)},
$$

and the non-negative integer

$$
c_{n}^{G}(A)=\operatorname{dim}_{F} P_{n}^{G}(A), n \geq 1,
$$

is the $n$th $G$-graded codimension of $A$. Our aim is to prove that $\exp ^{G}(A)=$ $\lim _{n \rightarrow \infty} \sqrt[n]{c_{n}^{G}(A)}$ exists and is an integer. Moreover, we shall relate such an integer to the dimension of a finite dimensional semisimple algebra related to $A$.

For every $n \geq 1$, write $n=n_{1}+\cdots+n_{s}$, a sum of non-negative integers, and let $P_{n_{1}, \ldots, n_{s}} \subseteq P_{n}^{G}$ be the space of multilinear graded polynomials in which the first $n_{1}$ variables have homogeneous degree $g_{1}$, the next $n_{2}$ variables have homogeneous degree $g_{2}$, and so on. Then $P_{n}^{G}$ is the direct sum of subspaces isomorphic to $P_{n_{1}, \ldots, n_{s}}$, for every choice of the integers $n_{1}, \ldots, n_{s}$. Moreover, such decomposition is inherited by $P_{n_{1}, \ldots, n_{s}} \cap \operatorname{Id}^{G}(A)$, and we define

$$
P_{n_{1}, \ldots, n_{s}}(A)=\frac{P_{n_{1}, \ldots, n_{s}}}{P_{n_{1}, \ldots, n_{s}} \cap \operatorname{Id}^{G}(A)} .
$$

If we let

$$
c_{n_{1}, \ldots, n_{s}}(A)=\operatorname{dim} P_{n_{1}, \ldots, n_{s}}(A),
$$

then, by checking dimensions we have

$$
c_{n}^{G}(A)=\sum_{n_{1}+\cdots+n_{s}=n}\left(\begin{array}{c}
n \\
n_{1}, \ldots, n_{s}
\end{array}\right) c_{n_{1}, \ldots, n_{s}}(A),
$$

where $\left(\begin{array}{c}n \\ n_{1}, \ldots, n_{s}\end{array}\right)=\frac{n !}{n_{1} ! \cdots n_{s} !}$ denotes the multinomial coefficient. In order to compute an upper and a lower bound for $c_{n}^{G}(A)$, it is enough to do so for $c_{n_{1}, \ldots, n_{s}}(A)$ and then apply (11).

The space $P_{n_{1}, \ldots, n_{s}}(A)$ is naturally endowed with a structure of $S_{n_{1}} \times \cdots \times S_{n_{s}}$ module in the following way. The group $S_{n_{1}} \times \cdots \times S_{n_{s}}$ acts on the left on $P_{n_{1}, \ldots, n_{s}}$ by permuting the variables of the same homogeneous degree; hence $S_{n_{1}}$ permutes the variables of homogeneous degree $g_{1} ; S_{n_{2}}$, those of homogeneous degree $g_{2}$; etc. Since $\operatorname{Id}^{G}(A)$ is invariant under this action, $P_{n_{1}, \ldots, n_{s}}(A)$ inherits a structure of $S_{n_{1}} \times \cdots \times S_{n_{s}}$-module, and we denote by $\chi_{n_{1}, \ldots, n_{s}}(A)$ its character.

If $\lambda$ is a partition of $n$, we write $\lambda \vdash n$. It is well-known that there is a one-toone correspondence between partitions of $n$ and irreducible $S_{n}$-characters. Hence if $\lambda \vdash n$, we denote by $\chi_{\lambda}$ the corresponding irreducible $S_{n}$-character. Now, if $\lambda(1) \vdash$ $n_{1}, \ldots, \lambda(s) \vdash n_{s}$, are partitions, then we write $\langle\lambda\rangle=(\lambda(1), \ldots, \lambda(s)) \vdash\left(n_{1}, \ldots, n_{s}\right)$ and we say that $\langle\lambda\rangle$ is a multipartition of $n=n_{1}+\cdots+n_{s}$.

Since char $F=0$, by complete reducibility, $\chi_{n_{1}, \ldots, n_{s}}(A)$ can be written as a sum of irreducible characters:

$$
\chi_{n_{1}, \ldots, n_{s}}(A)=\sum_{\langle\lambda\rangle \vdash n} m_{\langle\lambda\rangle} \chi_{\lambda(1)} \otimes \cdots \otimes \chi_{\lambda(s)},
$$

where $\langle\lambda\rangle=(\lambda(1), \ldots, \lambda(s)) \vdash\left(n_{1}, \ldots, n_{s}\right)$ is a multipartition of $n=n_{1}+\cdots+n_{s}$ and $m_{\langle\lambda\rangle} \geq 0$ is the multiplicity of $\chi_{\lambda(1)} \otimes \cdots \otimes \chi_{\lambda(s)}$ in $\chi_{n_{1}, \ldots, n_{s}}(A)$. 
Our first objective is to prove that the multiplicities in (2) are polynomially bounded.

Let $E=\left\langle e_{1}, e_{2}, \ldots \mid e_{i} e_{j}=-e_{j} e_{i}\right\rangle$ be the infinite dimensional Grassmann algebra over $F$ and let $E=E_{0} \oplus E_{1}$ be its standard $\mathbb{Z}_{2}$-grading. Now, if $B=$ $\bigoplus_{(g, i) \in G \times \mathbb{Z}_{2}} B_{(g, i)}$ is a $G \times \mathbb{Z}_{2}$-graded algebra, then $B$ has an induced $\mathbb{Z}_{2}$-grading, $B=B_{0} \oplus B_{1}$, where $B_{0}=\bigoplus_{g \in G} B_{(g, 0)}$ and $B_{1}=\bigoplus_{g \in G} B_{(g, 1)}$, and an induced $G$-grading, $B=\bigoplus_{g \in G} B_{g}$, where, for all $g \in G, B_{g}=B_{(g, 0)} \oplus B_{(g, 1)}$. Then one defines the Grassmann envelope of $B$ as $E(B)=\left(B_{0} \otimes E_{0}\right) \oplus\left(B_{1} \otimes E_{1}\right)$. Clearly $E(B)$ has a $G$-grading given by $E(B)=\bigoplus_{g \in G} E(B)_{g}$, where $E(B)_{g}=$ $\left(B_{(g, 0)} \otimes E_{0}\right) \oplus\left(B_{(g, 1)} \otimes E_{1}\right)$.

As in the ordinary case, the Grassmann envelope is an important object. In fact, by a result of Aljadeff and Belov ([2]), any variety of $G$-graded PI-algebras can be generated by the Grassmann envelope of a suitable finite dimensional $G \times \mathbb{Z}_{2}$-graded algebra.

Let $\mathcal{V}=\operatorname{var}^{G}(A)$ denote the variety of $G$-graded algebras generated by $A$ and define $\mathcal{V}^{*}=\left\{B=G \times \mathbb{Z}_{2}\right.$-graded algebra such that $\left.E(B) \in \mathcal{V}\right\}$. Then $\mathcal{V}^{*}$ is a variety (see [13, Theorem 3.7.5]).

Now, according to [9, Corollary 1], there exist integers $k \geq l \geq 0$ such that in (2) $\lambda(1), \ldots, \lambda(s) \in H(k, l)$, where $H(k, l)=\left\{\lambda \vdash n \mid \lambda_{k+1} \leq l\right\}$ is the infinite $k \times l$ hook.

Let $L$ be the relatively free $G \times \mathbb{Z}_{2}$-graded algebra of $\mathcal{V}^{*}$ on the $(k+l) s$ graded generators

$$
u_{\left(g_{i}, 0\right)}^{j}, v_{\left(g_{i}, 1\right)}^{p}, \quad 1 \leq i \leq s, 1 \leq j \leq k, 1 \leq p \leq l .
$$

Then $\mathcal{V}=\operatorname{var}^{G}(E(L))$ (see for instance [13, Theorem 4.8.2]).

Since $L$ is a finitely generated $G \times \mathbb{Z}_{2}$-graded PI-algebra, by [2, Theorem 1.1] there exists a finite dimensional $G \times \mathbb{Z}_{2}$-graded algebra $W$ such that $\operatorname{var}^{G \times \mathbb{Z}_{2}}(L)=$ $\operatorname{var}^{G \times \mathbb{Z}_{2}}(W)$. Moreover, $L$ is a homomorphic image of a relatively free graded algebra $T$ of such variety on $k s$ even generators and $l s$ odd generators.

The algebra $T$ can be constructed by "generic" elements as follows: fix a basis $\left\{a_{1} \ldots, a_{m}\right\}$ of $W$ of homogeneous elements. Let $\xi_{i}^{(t)}, 1 \leq i \leq m, 1 \leq t \leq(k+l) s$, be commutative variables and define $\xi_{\left(g_{i}, 0\right)}^{(t)}=\sum a_{i_{j}} \otimes \xi_{i_{j}}^{(t)}, \quad 1 \leq t \leq k$, where the sum runs over all $i_{j}$ such that $a_{i_{j}}$ is of homogeneous degree $\left(g_{i}, 0\right)$. Similarly define $\xi_{\left(g_{i}, 1\right)}^{(r)}=\sum a_{i_{j}} \otimes \xi_{i_{j}}^{(r)}, 1 \leq r \leq l$, where the $a_{i_{j}}$ are of homogeneous degree $\left(g_{i}, 1\right)$. Then $T$ is generated by the "generic" elements

$$
\xi_{\left(g_{i}, 0\right)}^{(t)}, \xi_{\left(g_{i}, 1\right)}^{(r)}, 1 \leq i \leq s, 1 \leq t \leq k, 1 \leq r \leq l
$$

Denote by $L_{n}$ the subspace of $L$ spanned by all products $w_{1} \cdots w_{i}, 1 \leq i \leq n$, where $w_{1}, \ldots, w_{i}$ run over the generators given in (3). Define $T_{n}$ accordingly on the relatively free generators given in (4). Since $L$ is a homomorphic image of $T$, $\operatorname{dim} L_{n} \leq \operatorname{dim} T_{n}$, and we compute an upper bound of this last dimension.

Notice that every monomial of degree at most $n$ in the generic elements in (4) belongs to $W \otimes F\left[\xi_{i}^{(t)}\right]_{n}$, where $F\left[\xi_{i}^{(t)}\right]_{n}$ is the subspace of polynomials of degree at most $n$ in the commutative variables $\xi_{i}^{(t)}, 1 \leq i \leq m, 1 \leq t \leq(k+l) s$. 
Since $\operatorname{dim} F\left[\xi_{i}^{(t)}\right]_{n}=\left(\begin{array}{c}(k+l) s m+n \\ n\end{array}\right) \leq(n+(k+l) s)^{(k+l) s m}$, we get that

$$
\operatorname{dim} L_{n} \leq \operatorname{dim} T_{n} \leq m(n+(k+l) s)^{(k+l) s m} \leq C n^{t},
$$

for some constants $C, t$.

We are now ready to prove the following.

Lemma 2.1. There exist constants $C, t$ such that for all $n \geq 1, m_{\langle\lambda\rangle} \leq C n^{t}$ in (2).

Proof. Suppose that there exists $n$ and $\langle\lambda\rangle \vdash n$ such that $m_{\langle\lambda\rangle}>C n^{t} \geq \operatorname{dim} L_{n}$, where $C$ and $t$ are defined in (5). Hence there exist $m_{\langle\lambda\rangle}=r$ irreducible $S_{n_{1}} \times$ $\cdots \times S_{n_{s}}$-modules $M_{1}, \ldots, M_{r} \in P_{n_{1}, \ldots, n_{s}}$ with character $\chi_{\lambda(1)} \otimes \cdots \otimes \chi_{\lambda(s)}$ and $\left(M_{1} \oplus \cdots \oplus M_{r}\right) \cap \operatorname{Id}^{G}(\mathcal{V})=0$. Now, as in [9, Lemma 4], we may take $M_{i}=$ $F\left(S_{n_{1}} \times \cdots \times S_{n_{s}}\right) h_{i}$, where, by eventually adding some empty sets of variables, we may assume that each $h_{i}$ is a polynomial in the homogeneous sets of variables $Y_{g_{1}}^{j}, \ldots, Y_{g_{s}}^{j}, Z_{g_{1}}^{p}, \ldots, Z_{g_{s}}^{p}, \quad i \leq j \leq k, 1 \leq p \leq l$, and $h_{i}$ is symmetric in each $Y_{g_{t}}^{j}$ and alternating in each $Z_{g_{t}}^{p}$. Since $\left(M_{1} \oplus \cdots \oplus M_{r}\right) \cap \operatorname{Id}^{G}(\mathcal{V})=0$, for every choice of $\alpha_{1}, \ldots, \alpha_{r} \in F$ not all zero, we have that $h=\sum_{i=1}^{r} \alpha_{i} h_{i} \notin \operatorname{Id}^{G}(\mathcal{V})$.

Let ${ }^{\sim}$ be the map defined in [9, Section 5]. Then, if we regard the variables of each set $Y_{g_{t}}^{j}$ as even variables and those of $Z_{g_{t}}^{p}$ as odd variables, we can construct the polynomials $\tilde{h}_{i}, 1 \leq i \leq r$. Then $\tilde{h}_{i}$ is symmetric on each $Y_{g_{t}}^{j}$ and on each $Z_{g_{t}}^{p}$.

For every $i$ and $j$, let $Y_{g_{i}}^{j}=\left\{y_{1, g_{i}}^{j}, \ldots, y_{m_{i}, g_{i}}^{j}\right\}$ and $Z_{g_{i}}^{j}=\left\{z_{1, g_{i}}^{j}, \ldots, z_{r_{i}, g_{i}}^{j}\right\}$. Define $S$ to be the relatively free $G$-graded algebra of the variety $\mathcal{V}$ on the graded generators

$$
\bar{y}_{p, g_{i}}^{j}, \bar{z}_{q, g_{i}}^{j}, \quad 1 \leq i \leq s, 1 \leq p \leq m_{i}, 1 \leq q \leq r_{i}, j=1,2, \ldots
$$

Then the algebra $Q=\left(S \otimes E_{0}\right) \oplus\left(S \otimes E_{1}\right)$ has a natural $G \times \mathbb{Z}_{2}$-grading and we can take its Grassmann envelope

$$
E(Q)=\left(S \otimes E_{0} \otimes E_{0}\right) \oplus\left(S \otimes E_{1} \otimes E_{1}\right) \subseteq S \otimes\left(E_{0} \otimes E_{0} \otimes E_{1} \otimes E_{1}\right) .
$$

Since $E_{0} \otimes E_{0} \otimes E_{1} \otimes E_{1}$ is commutative, $E(Q)$ and $S$ satisfy the same $G$-graded identities. Hence $E(Q) \in \mathcal{V}$, and so $Q \in \mathcal{V}^{*}$.

Now in each polynomial $\tilde{h}_{i}, 1 \leq i \leq r$, we identify the variables in each set $Y_{g_{i}}^{j}$ and in each set $Z_{g_{i}}^{j}$ and we let $\tilde{h}_{i}^{\circ}$ be the corresponding polynomial. Since $\operatorname{deg} \tilde{h}_{i}=n$, under the evaluation

$$
\varphi\left(Y_{g_{i}}^{j}\right)=u_{\left(g_{i}, 0\right)}^{j}, \varphi\left(Z_{g_{i}}^{p}\right)=v_{\left(g_{i}, 1\right)}^{p}, \quad 1 \leq i \leq s, 1 \leq j \leq k, 1 \leq p \leq l,
$$

we have that $\varphi\left(\tilde{h}_{i}^{\circ}\right) \in L_{n}$, for all $1 \leq i \leq r$.

Since by hypothesis $r>\operatorname{dim} L_{n}$, there exist scalars $\alpha_{1}, \ldots, \alpha_{r}$ not all zero, such that $\varphi\left(\sum_{i=1}^{r} \alpha_{i} \tilde{h}_{i}^{\circ}\right)=0$ in $L$. Recalling that $L$ is a relatively free algebra of $\mathcal{V}^{*}$, we obtain that $\tilde{h}^{\circ}=\sum_{i=1}^{r} \alpha_{i} \tilde{h}_{i}^{\circ} \in \operatorname{Id}^{G \times \mathbb{Z}_{2}}\left(\mathcal{V}^{*}\right)$. Now, $Q \in \mathcal{V}^{*}$, and so $\tilde{h}^{\circ} \in \operatorname{Id}^{G \times \mathbb{Z}_{2}}(Q)$.

If we consider the evaluation in $Q=\left(S \otimes E_{0}\right) \oplus\left(S \otimes E_{1}\right)$ defined by

$$
\begin{gathered}
\varphi\left(Y_{g_{i}}^{j}\right)=\bar{y}_{1, g_{i}}^{j} \otimes \alpha_{1}^{j}+\cdots+\bar{y}_{m_{i}, g_{i}}^{j} \otimes \alpha_{m_{i}}^{j}, \quad 1 \leq i \leq s, 1 \leq j \leq k, \\
\varphi\left(Z_{g_{i}}^{j^{\prime}}\right)=\bar{z}_{1, g_{i}}^{j^{\prime}} \otimes \beta_{1}^{j^{\prime}}+\cdots+\bar{z}_{r_{i}, g_{i}}^{j^{\prime}} \otimes \beta_{r_{i}}^{j^{\prime}}, \quad 1 \leq i \leq s, 1 \leq j^{\prime} \leq l,
\end{gathered}
$$

where $\alpha_{t}^{j}, \beta_{t}^{j^{\prime}}$ are disjoint monomials of $E$ of the correct homogeneous degree $\left(\alpha_{t}^{j}\right.$ of homogeneous degree 0 and $\beta_{t}^{j^{\prime}}$ of homogeneous degree 1$)$, we get that $\varphi\left(\tilde{h}^{\circ}\right)=0$. 
By computing explicitly we obtain $0=\varphi\left(\tilde{h}^{\circ}\right)=\varphi^{\prime}(\tilde{\tilde{h}}) \otimes \gamma$, where $0 \neq \gamma \in E$ and $\varphi^{\prime}$ is an evaluation such that

$$
\varphi^{\prime}\left(y_{p, g_{i}}^{j}\right)=\bar{y}_{p, g_{i}}^{j}, \varphi^{\prime}\left(z_{q, g_{i}}^{j^{\prime}}\right)=\bar{z}_{q, g_{i}}^{j^{\prime}},
$$

with $1 \leq p \leq m_{i}, 1 \leq \underset{q}{q} \leq r_{i}, 1 \leq j \leq k, 1 \leq j^{\prime} \leq l, 1 \leq i \leq s$. Since $\tilde{\tilde{h}}=h$, we obtain that $\varphi^{\prime}(h)=\varphi^{\prime}(\tilde{\tilde{h}})=0$.

Now, recall that the elements $\bar{y}_{p, g_{i}}^{j}, \bar{z}_{q, g_{i}}^{j}, j \geq 1$, generate the relatively free $G$ graded algebra of $\mathcal{V}$ of countable rank. Hence $\varphi^{\prime}(h)=0$ says that $h=\sum_{i=1}^{r} \alpha_{i} h_{i} \in$ $\operatorname{Id}^{G}(\mathcal{V})$, and this contradiction completes the proof.

Next we shall prove the existence of the $G$-exponent of $A$. Let $B$ be a finite dimensional $G \times \mathbb{Z}_{2}$-graded algebra such that $\operatorname{var}^{G}(A)=\operatorname{var}^{G}(E(B))$. By the Wedderburn-Malcev theorem [7] and the result in [21], we can write $B=B_{1} \oplus \cdots \oplus$ $B_{k}+J$, where $B_{1}, \ldots, B_{k}$ are $G \times \mathbb{Z}_{2}$-graded simple algebras and $J$ is the Jacobson radical of $B$. Recall that according to [9, Section 3], a semisimple subalgebra $D=D_{1} \oplus \cdots \oplus D_{h}$, where $D_{1}, \ldots, D_{h} \in\left\{B_{1}, \ldots, B_{k}\right\}$ are distinct, is admissible if $D_{1} J D_{2} J \cdots J D_{h} \neq 0$. Then one defines

$$
p=p(B)=\max (\operatorname{dim} D),
$$

where $D$ runs over all admissible subalgebras of $B$.

We shall prove that $p$ coincides with the $G$-exponent of $A$. In fact we have the following.

Theorem 2.2. Let $B$ be a finite dimensional $G \times \mathbb{Z}_{2}$-graded algebra over an algebraically closed field of characteristic zero. Then there exist constants $C_{1}>$ $0, C_{2}, k_{1}, k_{2}$ such that

$$
C_{1} n^{k_{1}} p^{n} \leq c_{n}^{G}(E(B)) \leq C_{2} n^{k_{2}} p^{n},
$$

where $p=p(B)$ is the integer defined in (6).

Proof. This theorem is proved in [9] for $G$ abelian, but the proof carries over to the non-abelian case by making the following changes.

In the computation of the upper bound $c_{n}^{G}(E(B)) \leq C_{2} n^{k_{2}} p^{n}$ one should use Lemma 2.1 above instead of [9, Remark 1].

For the computation of the lower bound $C_{1} n^{k_{1}} p^{n} \leq c_{n}^{G}(E(B))$ one should use Theorem 1.2 instead of [1, Lemma 18], concerning the construction of multialternating polynomials of arbitrarily large degree for finite dimensional $G$-graded simple algebras. We should point out that while the polynomial constructed in [1, Lemma 18] depends on a parameter $t$, the one constructed in Theorem 1.2 depends on $s$ parameters $t_{1}, \ldots, t_{s}$ (each corresponding to a homogeneous component of the algebra) and these parameters are squeezed between $2 t$ and $2|G| t$. Then one notices that in [9, Section 6] the proofs are carried over for each homogeneous component separately. This completes the proof of the theorem.

Since graded codimensions do not change by extension of the base field, we get the following.

Theorem 2.3. Let $G$ be a finite group and $A$ be a $G$-graded PI-algebra over any field $F$ of characteristic zero. Then $\exp ^{G}(A)=\lim _{n \rightarrow \infty} \sqrt[n]{c_{n}^{G}(A)}$ exists and is an integer. 


\section{REFERENCES}

[1] E. Aljadeff, A. Giambruno and D. La Mattina, Graded polynomial identities and exponential growth, J. Reine Angew. Math. 650 (2011), 83-100. MR2770557

[2] E. Aljadeff and A. Kanel-Belov, Representability and Specht problem for G-graded algebras, Adv. Math. 225 (2010), 2391-2428. MR2680170

[3] Y. A. Bahturin and V. Drensky, Graded polynomial identities of matrices, Linear Algebra Appl. 357 (2002), 15-34. MR1935223(2003k:16034)

[4] Y. A. Bahturin, S. K. Sehgal and M. V. Zaicev, Finite-dimensional simple graded algebras, Sb. Math. 199 (2008), no. 7, 965-983. MR2488221 (2009k:16080)

[5] A. Berele and A. Regev, Hook Young diagrams with applications to combinatorics and to representations of Lie superalgebras, Adv. Math. 64 (1987), 118-175. MR884183 (88i:20006)

[6] A. Berele and A. Regev, Exponential growth for codimensions of some p.i. algebras, J. Algebra 241 (2001), 118-145. MR.1838847 (2002k:16046)

[7] C. W. Curtis and I. Reiner, Representation theory of finite groups and associative algebras, Wiley Classics Library, John Wiley \& Sons, Inc., New York, 1988. MR1013113 (90g:16001)

[8] E. Formanek, A conjecture of Regev about the Capelli polynomial, J. Algebra 109 (1987), 93-114. MR898339(88i:16019)

[9] A. Giambruno and D. La Mattina, Graded polynomial identities and codimensions: computing the exponential growth, Adv. Math. 225 (2010), 859-881. MR2671182 (2011f:16047)

[10] A. Giambruno and A. Regev, Wreath products and P.I. algebras, J. Pure Applied Algebra 35 (1985), 133-149. MR775466 (86e:16027)

[11] A. Giambruno and M. Zaicev, On codimension growth of finitely generated associative algebras, Adv. Math. 140 (1998), 145-155. MR.1658530 (99k:16049)

[12] A. Giambruno and M. Zaicev, Exponential codimension growth of PI algebras: an exact estimate, Adv. Math. 142 (1999), 221-243. MR1680198(2000a:16048)

[13] A. Giambruno and M. Zaicev, Polynomial Identities and Asymptotic Methods, Mathematical Surveys and Monographs, Vol. 122, AMS, Providence, R.I., 2005. MR2176105|(2006g:16054)

[14] G. James and A. Kerber, The Representation Theory of the Symmetric Group, Encyclopedia of Mathematics and its Applications, Vol. 16, Addison-Wesley, London, 1981. MR644144 (83k:20003)

[15] A. Kemer, Ideals of Identities of Associative Algebras, AMS Translations of Mathematical Monographs, Vol. 87, Providence, R.I., 1991. MR1108620 (92f:16031)

[16] D. S. Passman, Infinite Crossed Products, Academic Press, San Diego, CA, 1989. MR979094 (90g:16002)

[17] D. Quinn, Group-graded rings and duality, Trans. Amer. Math. Soc. 292 (1985), no. 1, 155167. MR805958 (87d:16002)

[18] A. Regev, Existence of identities in $A \otimes B$, Israel J. Math. 11 (1972), 131-152. MR0314893 $(47: 3442)$

[19] A. Regev, Asymptotic values for degrees associated with stripes of Young diagrams, Adv. in Math. 41 (1981), 115-136. MR625890(82h:20015)

[20] I. Sviridova, Identities of pi-algebras graded by a finite abelian group, Comm. Algebra 39 (2011), 3462-3490. MR2845581 (2012h:16046)

[21] E. Taft, Orthogonal conjugacies in associative and Lie algebras, Trans. Amer. Math. Soc. 113 (1964), 18-29. MR0163930 (29:1229)

Department of Mathematics, Technion-Israel Institute of Technology, Haifa 32000 , ISRAEL

E-mail address: aljadeff@tx.technion.ac.il

Dipartimento di Matematica e Informatica, Università di Palermo, Via Archirafi 34, 90123 PAlermo, ItAly

E-mail address: antonio.giambruno@unipa.it 